\title{
Biomass carbon materials derived from macadamia nut shells for high-performance supercapacitors
}

\author{
XIAOWEI LU, KAIXIONG XIANG, WEI ZHOU, YIRONG ZHU and HAN CHEN* \\ School of Metallurgical and Materials Engineering, Hunan University of Technology, Zhuzhou 412007, Hunan, \\ People's Republic of China \\ *Author for correspondence (lzdxnchh@126.com)
}

MS received 13 October 2017; accepted 3 February 2018; published online 28 November 2018

\begin{abstract}
The new biomass carbonaceous materials were obtained from macadamia nut shells (MNS) by immersion method and high-temperature activation (MNSCA) for high-performance supercapacitors. The morphologies and microstructures are investigated by X-ray diffractometer, Raman spectrometer, scanning electron microscopy and transmission electron microscopy. The experimental results show that the obtained activated carbon (MNSCA) exhibits perfect porous structure filled with more micropores and mesopores. MNSCA displays high specific surface area of $1057 \mathrm{~m}^{2} \mathrm{~g}^{-1}$. The porous carbon delivers an impressive specific capacitance of $325.7 \mathrm{Fg}^{-1}$ and has no capacitance loss at the current density of $2 \mathrm{Ag}^{-1}$ after 10,000 cycles, which demonstrates the excellent cycle stability and high specific capacitance. The biomass carbonaceous materials derived from MNS can be expected for the widespread application of supercapacitors.
\end{abstract}

Keywords. Supercapacitor; activated carbon; porous structure; macadamia nut shells.

\section{Introduction}

With a fast growing market for the energy storage devices such as hybrid electric vehicles, medical equipments and portable electric equipments, there is a critical requirement to develop a new sustainable and renewable energy [1,2]. Electrochemical capacitors can be extensively applied in the energy storage devices owing to its excellent recharge/charge performance, high power capability, extraordinary cycle stability, nontoxic nature [3-6]. There is an urgent demand for new biomass carbonaceous materials with unique structures for applications as the supercapacitor electrodes. However, the practical utilization of supercapacitors can be limited due to low-energy density. Consequently, it is an urgent problem to enhance the specific capacitance and cycle stability of electrode materials for supercapacitors [5,7-12].

In recent years, the biomass-derived pyrolytic carbon materials were applied for supercapacitors, such as willow catkins [13], kenaf stem [14], banana peels [15-17], bean dregs [18], watermelon pulp [19], corn grains [20-22], etc. The biomass carbon resources derived from solid waste have the characteristics of abundance, low-cost, simple preparation and can reduce environmental pollution. Hence, natural biomass materials are believed to replace the conventional fossil fuel in the future and will play an important role in energy-storage field. Whereas, the electrochemical performance of the biomass-derived carbons was not completely desirable, especially in low capacity and/or poor-cycling performance, and these material morphologies are affected by original morphologies of the biomass waste and the impurity has a great effect on material property. Therefore, the morphology modifications for these materials are the most important and efficient methods to improve the performance of biomass carbon materials.

In this work, macadamia nut shells (MNS) were selected as the promising biomass precursors for the supercapacitor electrode, and the porous-activated carbons were prepared by a facile-activated hydrothermal method and high-temperature activation treatment with $\mathrm{KOH}$. The structural characteristics of activated carbon, such as pore structure, surface morphologies, pore size distribution and its electrochemical performance were systematically investigated.

\section{Experimental}

\subsection{Materials preparation}

The MNS raw materials were obtained from our household waste. The activated carbons were prepared via the following facile procedures. Firstly, the MNS were ground into powder by ball-milling and these were washed with deionized water and dilute $\mathrm{HCl}$ to remove some impurities, and then dried overnight in an electric drying oven. Secondly, $2 \mathrm{~g}$ MNS powder and $6 \mathrm{~g} \mathrm{KOH}$ were mixed in $60 \mathrm{ml}$ deionized water, transferred into a Telfon-lined stainless autoclave, and put it into an electric oven at $180^{\circ} \mathrm{C}$ for $12 \mathrm{~h}$. Then, the obtained product was filtered and dried in an electric oven. Thirdly, the obtained product was put into a tubular furnace and heated to $800^{\circ} \mathrm{C}$ for $2 \mathrm{~h}$ in $\mathrm{Ar}$ flow. In the end, the activated carbons were 
alternately washed with $\mathrm{HNO}_{3}(15 \%)$ and deionized water, and then dried in an electric oven. The obtained activated carbon by the above method was denoted as MNSCB (carbon materials of Group B sample derived from macadamia nut shell).

Additionally, the MNS powders were soaked in $\mathrm{KOH}$ solution for five days with the weight ratio of $\mathrm{MNS} / \mathrm{KOH}=$ 1/3. Similarly, the soaked MNS powder was calcined under $\mathrm{Ar}$ flow at $800^{\circ} \mathrm{C}$ for $2 \mathrm{~h}$. Then, the final obtained activated carbon via this immersion method was denoted as MNSCA (the carbon materials of Group A sample derived from macadamia nut shell). As a contrast, MNS powders were directly calcined to pyrolytic carbons untreated with $\mathrm{KOH}$, the control carbon sample denoted as MNSC.

\subsection{Materials characterization}

X-ray diffraction patterns (XRD) were operated on a Siemens D5000 using the $\mathrm{CuK} \alpha$ radiation of $(1=1.5418 \AA)$. The special morphologies of the samples were observed on the field emission scanning electron microscopy (FESEM) (JSM-6700F, JEOL, Tokyo, Japan). Transmission electron microscopy (TEM) images were taken on the JEOL 2010 microscope operating at $200 \mathrm{keV}$. Raman spectroscopy analysis was obtained by using a Renishaw Invia system with a laser wavelength of $514 \mathrm{~nm}$. Nitrogen sorption analyses were evaluated with a Micromeritics ASAP 2020 adsorption analyzer via the Brunauer-Emmett-Teller (BET).

\subsection{Electrochemical testing}

The electrochemical capacitance of the activated carbon was evaluated in a three-electrode system and operated on a CHI 660E electrochemical work station (Shanghai Chen Hua Precision Instruments Co.) under room temperature. The working electrode was prepared by mixing the $80 \mathrm{wt} \%$ active materials (graphene-like carbon), $10 \mathrm{wt} \%$ acetylene black and $10 \mathrm{wt} \%$ polyvinylidene fluoride (PVDF) with $\mathrm{N}$ methylpyrrolidone (NMP) as a solvent. Then, the obtained mixtures were painted onto nickel foam, and the as-prepared sample was dried at $80^{\circ} \mathrm{C}$ overnight in a vacuum oven and acted as the working electrode. Then, the three-electrode system containing Pt slice as the counter electrode and saturated calomel electrode (SCE) as the reference electrode can be used to test the electrochemical performance in $2 \mathrm{~mol}^{-1}$ $\mathrm{KOH}$ aqueous electrolyte solution, and the electrochemical performances of samples were investigated via using cyclic voltammogram (CV) curves, galvanostatic charge/discharge and electrochemical impedance spectroscopy (EIS). The EIS measurements were performed for the working electrode in a frequency range from $0.01 \mathrm{~Hz}$ to $100 \mathrm{kHz}$. The $\mathrm{CV}$ characteristic and galvanostatic charge/discharge performance were tested in the CHI660E electrochemical work station with the voltage range between -1.0 and $0.0 \mathrm{~V}$.

\section{Results and discussion}

Figure 1 depicts the SEM and TEM observations of activated carbons MNSCA, MNSCB and MNSC. It can clearly be seen
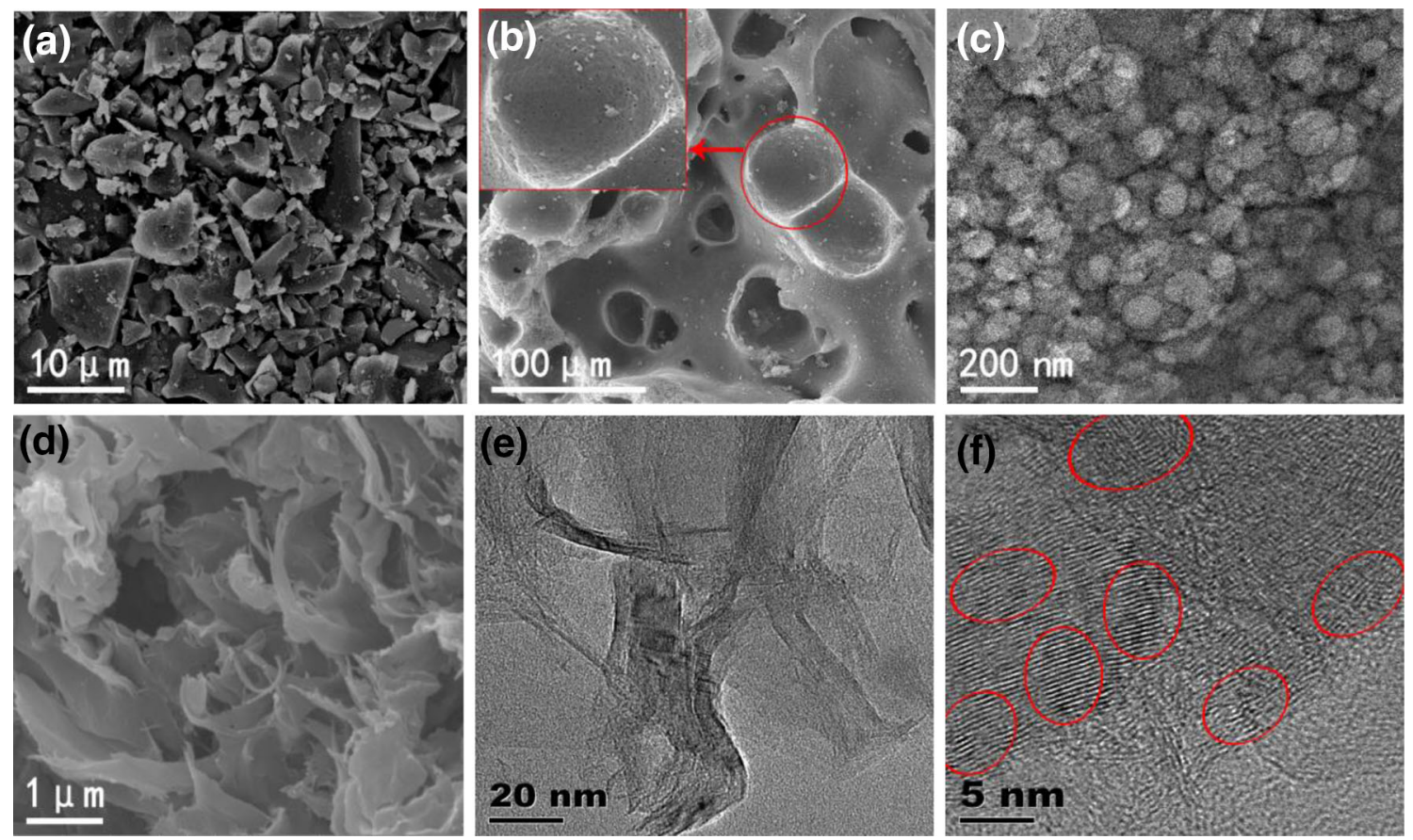

Figure 1. (a) SEM images of MNSC; (b, c) SEM and TEM images of MNSCA; and (d, e, f) SEM and TEM images of MNSCB. 

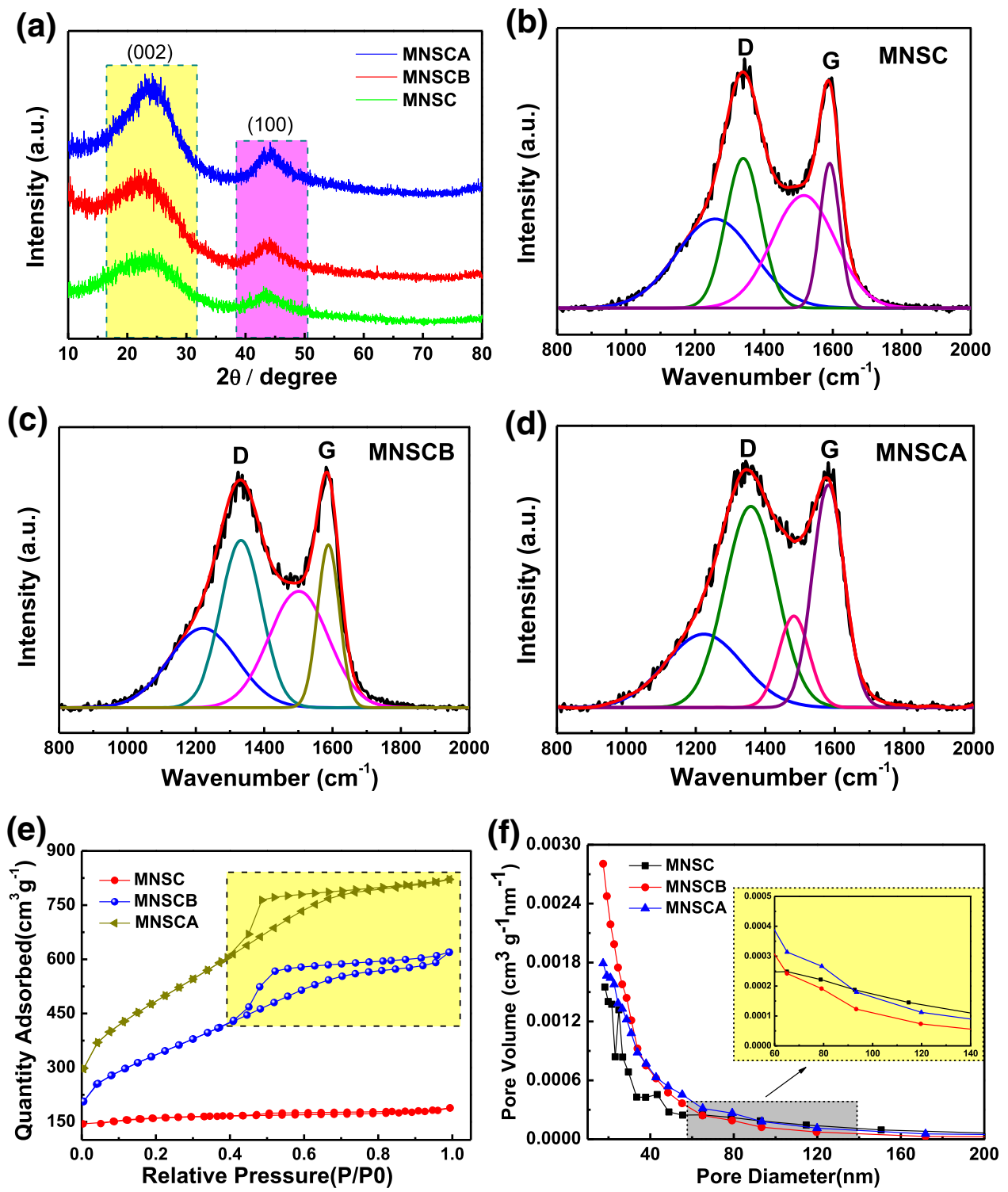

Figure 2. (a) XRD patterns; (b, c, d) Raman spectra; (e) nitrogen adsorption-desorption isotherms and (f) pore-size distributions obtained for MNSC, MNSCB and MNSCA.

from figure 1a that MNSC sample untreated with $\mathrm{KOH}$ displays clumpy structure without obvious pore structure. SEM (figure 1b) and TEM (figure 1c) observations of MNSCA show that the sample possesses irregular shapes and different sizes of pores with the size ranging from a few to tens of micrometres. As observed in figure $1 \mathrm{~b}$, it is noteworthy that the MNSCA has not only micrometric pores on the surface, but also interconnected small pores within the large micrometric pores. SEM and TEM images of activated carbons (MNSCB) (figure 1d-f) show that ultrathin carbon layers like graphene, accumulate to form fluffy and porous foamstructure. The ultrathin carbon layers can be clearly observed in figure 1d. The high-resolution TEM image (figure 1f) displays that graphitized carbons are embedded in amorphous carbon layer. The porous-activated carbons were caused by the corrosion of $\mathrm{KOH}$ under the high temperature of $800^{\circ} \mathrm{C}$, and the hydrothermal treatment further loosens the carbon materials microstructure [23]. The porous-activated structure is extremely important for supercapacitors owing to provide the interconnected channels, which enhance the transmittability of ions in interior electrolyte [24].

Figure 2a shows the XRD patterns of activated carbons. All three samples exhibit no sharp peaks and the broad peak (002) at about $23^{\circ}$, which represents the amorphous carbon, and the weaker peak (100) reflections appearing about $43^{\circ}$ arose from crystal structure derived from graphite microcrystalline, which depends on materials and craftsmanship [25]. Additionally, the spacing $\left(\mathrm{d}_{002}\right)$ values of intergraphene layer for MNSCB $(0.3657 \mathrm{~nm})$ and MNSCA $(0.3845 \mathrm{~nm})$ are larger than MNSC (0.3493 nm), as shown in table 1, it 
Table 1. Structure and textural properties of macadamia nut shells.

\begin{tabular}{lccccccc}
\hline Sample & $d_{002}(\mathrm{~nm})$ & $L_{\mathrm{a}}(\mathrm{nm})$ & $L_{\mathrm{c}}(\mathrm{nm})$ & $I_{\mathrm{D}} / I_{\mathrm{G}}$ & $S_{\mathrm{BET}}\left(\mathrm{m}^{2} \mathrm{~g}^{-1}\right)$ & Pore volume $\left(\mathrm{cm}^{3} \mathrm{~g}^{-1}\right)$ & Average pore diameter $(\mathrm{nm})$ \\
\hline MNSC & 0.3493 & 2.49 & 0.78 & 1.09 & 247 & 0.1275 & 14.87 \\
MNSCB & 0.3657 & 2.95 & 0.95 & 1.18 & 875 & 0.1846 & 21.75 \\
MNSCA & 0.3845 & 3.37 & 1.13 & 1.22 & 1057 & 1.1357 & 18.94 \\
\hline
\end{tabular}

indicates that the (002) peaks of MNSCB and MNSCA have a more well-developed intensity and the turbostratic disorder arises during the $\mathrm{KOH}$ activation process. The graphitization degree for activated carbons was confirmed from the Raman spectra (figure $2 b-d$ ), which gives a further evidence for the microstructures of carbon materials. Three samples mainly display two obvious peaks located at around 1350 and $1590 \mathrm{~cm}^{-1}$. The G-band appearing at $1590 \mathrm{~cm}^{-1}$ is attributed to the $\mathrm{sp}^{2}$ graphitic structure, while the peak of the D-band near $1350 \mathrm{~cm}^{-1}$ is related to disordered structure for amorphous carbon [26]. The relative intensity ratio of the D-band and G-band $\left(I_{\mathrm{D}} / I_{\mathrm{G}}\right)$ represents the graphitization degree of carbon materials [27]. As table 1 shows the $I_{\mathrm{D}} / I_{\mathrm{G}}$ values of MNSC, MNSCB and MNSCA are determined to be $1.09,1.18$ and 1.22 , respectively. It can be clearly seen that the $I_{\mathrm{D}} / I_{\mathrm{G}}$ ratio of the MNSC is smaller than MNSCB and MNSCA. These observations confirmed that the activation treatment with $\mathrm{KOH}$ leads to a lower graphitic degree. The Raman results are consistent with XRD analysis presented in figure 2a.

The more detailed structure characteristics of activated carbons were investigated by nitrogen adsorption-desorption isotherms shown in figure 2e. It clearly displays that MNSC sample shows its nonporous structure, both MNSCB and MNSCA show typical characteristics of type-IV sorption isotherms with a much higher $\mathrm{N}_{2}$ sorption capacity than MNSC, illustrating porous structure [28]. The typical characteristic of the type-IV has a hysteresis loop extending from $P / P_{0}=0.4-1.0$ as shown in figure $2 \mathrm{e}$, owing to capillary condensation in mesoporous carbon [29]. The porosity of the activated carbons is related to activation effect for $\mathrm{KOH}$ activating agent under high temperature. The corresponding pore-size distribution is shown in figure $2 \mathrm{f}$. MNSCB and MNSCA are mainly made of abundant mesopores and small amount of micropores and macropores. In addition, as table 1 shows that the specific Brunauer-Emmett-Teller (BET) surface area of MNSC, MNSCB and MNSCA are 247, 875 and $1057 \mathrm{~m}^{2} \mathrm{~g}^{-1}$, respectively. It can be obviously seen that the specific BET surface area of MNSCB and MNSCA are larger than MNSC. The activation temperature has a significant impact on the pore structure of MNSCB and MNSCA, as the $\mathrm{KOH}$ activation process was deepened at high-activation temperature, more micropores and mesopores were generated, resulting in higher surface area and total pore volume as shown in table 1. The large specific surface areas, abundant mesopores and high pore volumes play a significant role on the charge storage, transportation and electrolyte penetration, the reason for this is that these structures are favourable for supplying multiple channels and reducing the diffusion distance charges to the interior [30].

To evaluate the electrochemical capacitive properties of the activated carbon as supercapacitor electrodes materials, the CV and galvanostatic charge/discharge measurements were carried out in $2 \mathrm{~mol}^{-1} \mathrm{KOH}$ aqueous electrolyte solution. Figure 3 a displays $\mathrm{CV}$ curves of three samples MNSC, MNSCB and MNSCA at scan rate of $20 \mathrm{mV} \mathrm{s}^{-1}$. It is observed that all the curves of three sample electrodes exhibit rectangular-shape curves with obvious hump at low-potential region. Nevertheless, the CV curves of MNSCA and MNSCB are closer to the rectangle shape than MNSC, and the CV curves of MNSCA are closest to the rectangle, indicating the ideal double-layer capacitance characteristic [31]. Figure 3c and e shows the CV curves of activated samples MNSCB and MNSCA at scan rates from 5 to $100 \mathrm{mV} \mathrm{s}^{-1}$ between -1.0 and $0.0 \mathrm{~V}$. The CV curves of MNSCA and MNSCB can keep a typical quasi-rectangular shape with minor distortions even the scan rate was increased to $100 \mathrm{mV} \mathrm{s}^{-1}$, which indicates that MNSCA and MNSCB sample electrodes have unique EDLC performance and better charge-transfer efficiency. Figure $3 \mathrm{~b}$ depicts the galvanostatic charge/discharge curves of three samples MNSC, MNSCB and MNSCA at current density of $1 \mathrm{~A} \mathrm{~g}^{-1}$. It can be concluded that the specific capacitance of the MNSC, MNSCB and MNSCA electrodes were calculated from the discharging curves in figure $3 \mathrm{~b}$, and the results are determined to be 171.2, 250.6 and $325.7 \mathrm{~F} \mathrm{~g}^{-1}$ at the current density of $1 \mathrm{~A} \mathrm{~g}^{-1}$, respectively. As a consequence, it can be clearly seen that the specific capacitance values of the MNSCA are highest among them as shown in figure $3 b$, which is superior to the capacitance of the polypyrrole-derived microporous carbon nanospheres and nitrogen-doped carbon nanocages derived from polyaniline [32,33]. Moreover, figure $3 \mathrm{~d}$ and $\mathrm{f}$ depicts galvanostatic charge/discharge curves of activated samples MNSCB and MNSCA at various current densities. It can be seen that all curves of MNSCB and MNSCA are almost symmetrical and linear at increased current densities ranging from 1 to $20 \mathrm{~A} \mathrm{~g}^{-1}$, and the capacitances are reduced correspondingly according to the discharging time, which is a typical characteristic of an ideal electrochemical double-layer capacitor with good electrochemical reversibility. The impressive capacitive properties are directly related to the high-specific surface area and the unique structure features of the activated carbons. 

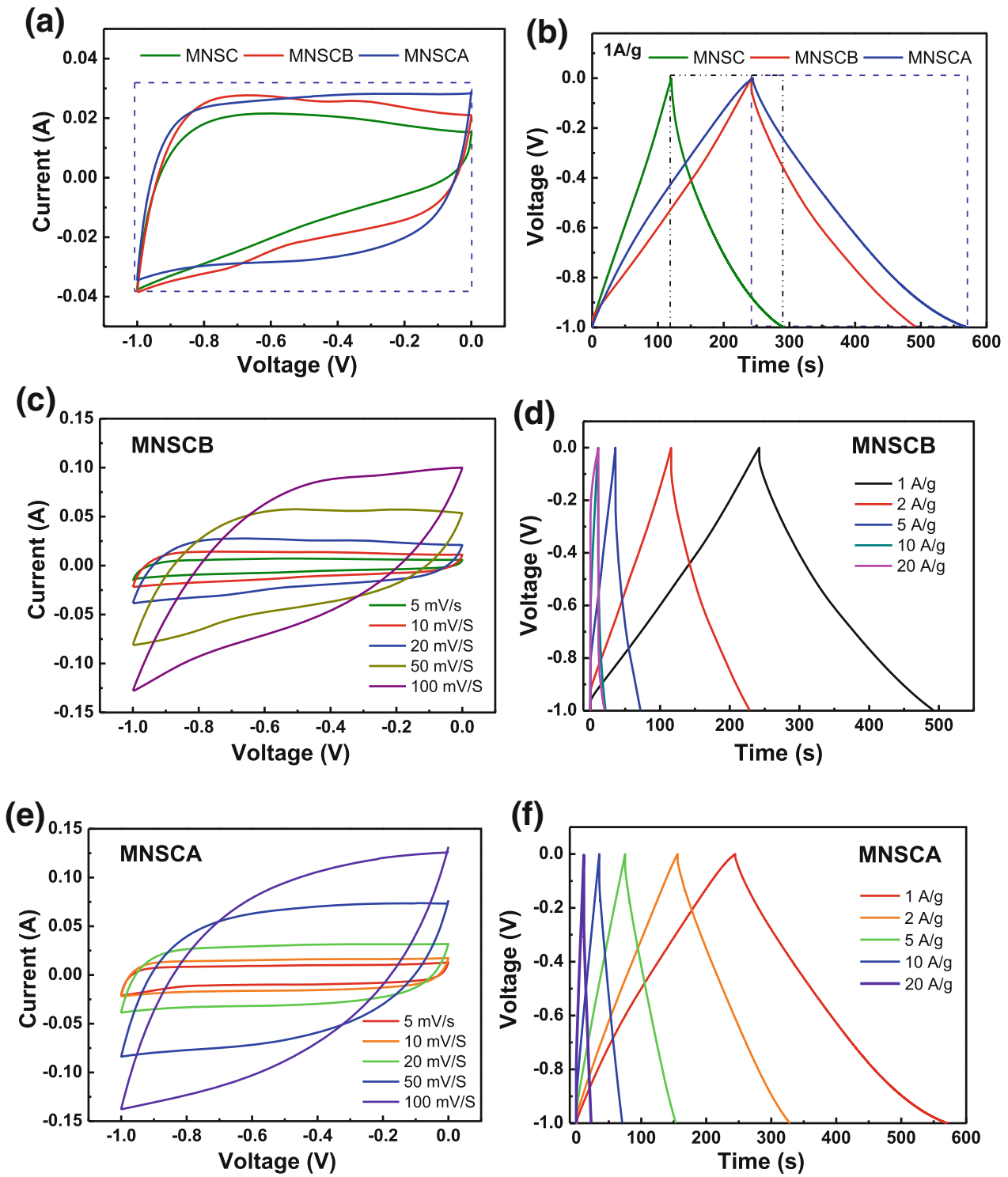

Figure 3. (a) Cyclic voltammetry curves of activated samples MNSC, MNSCB and MNSCA at scan rate of $20 \mathrm{mV} \mathrm{s}^{-1}$; (b) galvanostatic charge/discharge curves of the three samples at current density of $1 \mathrm{~A} \mathrm{~g}^{-1}$; cyclic voltammetry and galvanostatic charge/discharge curves of activated samples (c, d) MNSCB and (e, f) MNSCA.

To further evaluate the suitability of the MNSC, MNSCB and MNSCA electrodes for practical applications, the cycle life of three sample electrodes was determined by measuring galvanostatic charge/discharge at a current density of $2 \mathrm{~A} \mathrm{~g}^{-1}$ for 10,000 cycles as shown in figure $4 \mathrm{a}$. It is clear that the capacitance retentions of MNSC, MNSCB and MNSCA are determined to be $86.7,93.1$ and $97.2 \%$ after withstanding 10,000 charge/discharge cycles, respectively. Hence, the retentions of three electrodes are $\geq 86.7 \%$ of their initial capacitance. In addition, the MNSCA electrode delivers not only the highest specific capacitance, but also the impressive capacitance retention $(97.2 \%)$ and the retention is greatest among them, indicating the remarkable cycle stability, which is superior to the capacitance retention of the naturally three-dimensional laminated porous carbon network structured short nano-chains bridging nanospheres $(93.52 \%$ for CAs-800) [34]. To highlight the electrochemical performance advantage, this work (MNSCA) was compared with some biomass carbonaceous materials for supercapacitors, the electrodes are investigated by the galvanostatic chargedischarge experiments at various current densities as shown in figure $4 \mathrm{~b}$. It can be seen clearly that the specific capacitance of MNSCA is superior to those reported previously in literature [35-37].

Figure 5 shows that the EIS of the MNSC, MNSCB and MNSCA in the frequency range from $0.01 \mathrm{~Hz}$ to $0.1 \mathrm{MHz}$ 
(a)

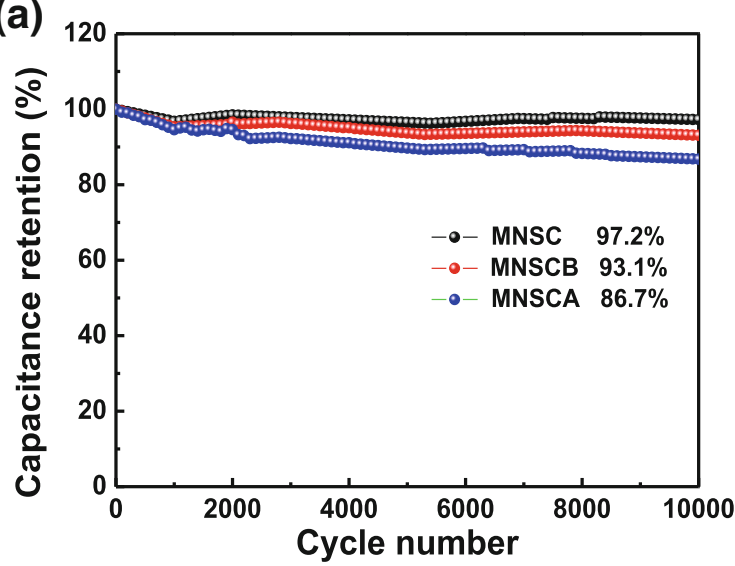

(b)

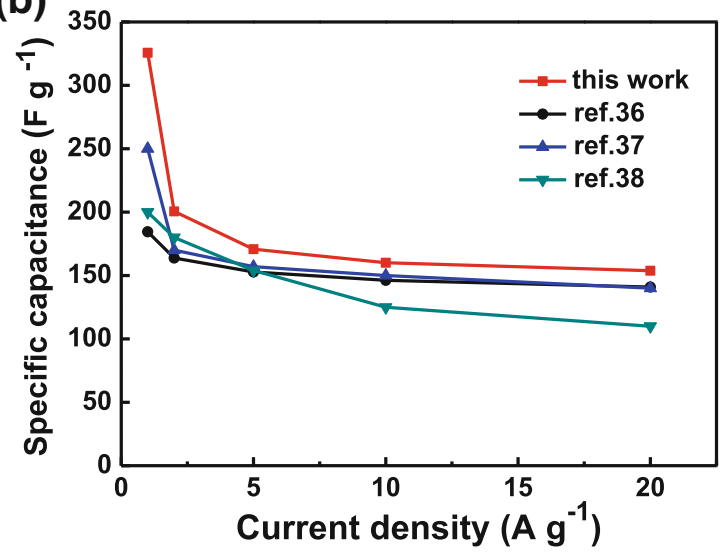

Figure 4. (a) The cycle life of three sample electrodes was determined by measuring galvanostatic charge/discharge at a current density of $2 \mathrm{~A} \mathrm{~g}^{-1}$ for 10,000 cycles; (b) comparison on electrochemical performances of biomass carbonaceous materials for supercapacitors.

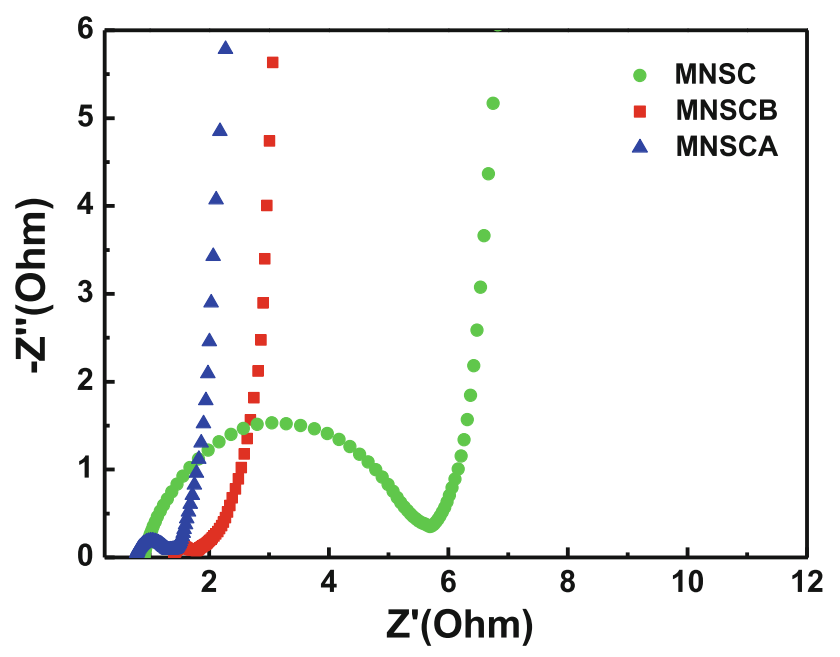

Figure 5. Electrochemical impedance spectra of the three samples MNSC, MNSCB and MNSCA in the frequency range from $0.01 \mathrm{~Hz}$ to $0.1 \mathrm{MHz}$.

under open circuit potential. It is seen that the entire EIS of three electrodes appear in a semicircle in the high-frequency region and nearly in a vertical slope in the low-frequency region $[38,39]$. The small semicircle diameter of the MNSCB and MNSCA (nearly $1 \Omega$ ) is smaller than MNSC (nearly $5 \Omega$ ), indicating the good ionic conductivity due to high electrical conductivity of electrode materials. At the lowfrequency region, a straight line is obtained due to the accumulation of electrolyte ions at the bottom of pores. The result indicates that the MNSCB and MNSCA electrode materials have low ionic resistance inside the porous structure of the activated carbons.

\section{Conclusions}

New biomass carbonaceous materials derived from MNS were prepared via the hydrothermal method and hightemperature activation. The activated carbons (MNSCA) exhibit an impressive specific capacitance of $325.7 \mathrm{~F} \mathrm{~g}^{-1}$ at the current density of $1 \mathrm{~A} \mathrm{~g}^{-1}$ with capacity retention of $97.2 \%$ after 10,000 cycles. The excellent performance is mainly due to abundant pores and porous foam-structure and high specific surface area. Therefore, the new biomass carbonaceous materials derived from MNS are a promising candidate for developing high-performance supercapacitor.

\section{Acknowledgements}

This work was supported by the National Nature Science Foundation of China (51772090, 51572079), Natural Science Foundation of Hunan Province (2016JJ5008, 2016JJ5041) and Hunan Provincial Education Department (16A055).

\section{References}

[1] Simon P and Gogotsi Y 2008 Nat. Mater. 7845

[2] Pech D, Durou M H, Huang P, Mochalin V, Gogotsi Y, Taberna P et al 2010 Nat. Nanotechnol. 5651

[3] Liu C, Yu Z, Neff D, Zhamu A and Zhamu B Z 2010 Nano Lett. 104863

[4] Li H B, Yu M H, Lu X H, Liu P, Liang Y, Xiao J et al 2014 ACS Appl. Mat. Interfaces 6745

[5] Xie K, Qin X T, Wang X Z, Wang Y N, Tao H S, Wu Q et al 2012 Adv. Mater. 24347

[6] Izadi-Najafabadi A, Yasuda S, Kobashi K, Yamada T, Futaba D N, Hatori H et al 2010 Adv. Mater. 22235 
[7] Zou Y and Wang Y 2013 Chem. Eng. J. 229183

[8] Miller J R and Simon P 2008 Sci. Mag. 321651

[9] Zhai Y P, Dou Y Q, Zhao D Y, Fulvio P F, Mayes R T and Dai S 2011 Adv. Mater. 234828

[10] Kim H, Fortunato M E, Xu H X, Bang J H and Suslick K S 2011 J. Phys. Chem. C $\mathbf{1 1 5} 20481$

[11] Lei Z B, Christov N and Zhao X S 2011 Energy Environ. Sci. 4 1866

[12] Zhu Y W, Murali S, Stoller M D, Ganesh K J, Cai W W, Ferreira $\mathrm{P}$ J et al 2011 Science 321537

[13] Wang K, Zhao N, Lei S, Lei S W, Yan R, Tian X D et al 2015 Electrochim. Acta 1661

[14] Wang L, Zheng Y L, Zhang Q Y, Zuo L, Chen S L, Chen S H et al 2014 RSC Adv. 451072

[15] Lv Y K, Gan L H, Liu M X, Xiong W, Xu Z J, Zhu D Z et al 2012 J. Power Sources 209152

[16] Emaga T H, Bindelle J, Agneesens R, Buldgen A, Wathelet B and Paquot M 2011 Trop. Anim. Health Prod. 43 171

[17] Subramanian V, Luo C, Stephan A M, Nahm K S, Thomas S and Wei B Q 2007 J. Phys. Chem. C 1117527

[18] Ruan C P, Ai K L and Lu L H 2014 RSC Adv. 430887

[19] Wu X L, Wen T, Guo H L, Yang S B, Wang X K and Xu A W 2013 ACS Nano 73589

[20] Balathanigaimani M S, Shim W G, Lee M J, Kim C, Lee J W and Moon H 2008 Electrochem. Commun. 10868

[21] Wang S B, Xiao C L, Xing Y L, Xu H Z and Zhang S C 2015 J. Mater. Chem. A 36742

[22] Chen M, Yu C, Liu S H, Fan X M, Zhao C T, Zhang X et al 2015 Nanoscale 71791

[23] Wang H L, Xu Z W, Kohandehghan A, Zhi L, Cui K, Tan X H et al 2013 ACS Nano 75131
[24] Wang L, Mu G, Tian C G, Sun L, Zhou W, Yu P et al 2013 ChemSusChem 6880

[25] Arrebola J C, Caballero A, Hernán L, Morales J, Marín M O and Serrano V G 2010 J. Electrochem. Soc. 157 A791

[26] Sadezky A, Muckenhuber H, Grothe H, Niessner R and Poschl U 2005 Carbon 431731

[27] Mohiuddin T M G, Lombardo A, Nair R R, Bonetti A, Savini G, Jalil R et al 2009 Phys. Rev. B 79205433

[28] Xing W B, Dunlap R A and Dahn J R 1998 J. Electrochem. Soc. 14562

[29] Nakagawa K, Mukai S R, Tamura K and Tamon H 2007 Chem. Eng. Res. Des. 851331

[30] Jiang J, Li Y Y, Liu J P, Huang X T, Yuan C Z and Lou X W 2012 Adv. Mater. 245166

[31] Huang H, Luo G, Xu L, Lei C, Tang Y, Tang S et al 2015 Nanoscale 72060

[32] Su F B, Poh C K, Chen J S, Xu G W, Wang D, Li Q et al 2011 Energy Environ. Sci. 4717

[33] Tan Y M, Xu C F, Chen G X, Liu Z H, Ma M, Xie Q J et al 2013 ACS Appl. Mat. Interfaces 52241

[34] Wang C, Xiong Y, Wang H W, Jin C D and Sun Q F 2017 J. Mater. Chem. A 515759

[35] Yang Y C, Shi W, Zhang R H, Luanx C H, Zeng Q, Wang C et al 2016 Electrochim. Acta 204100

[36] Zhu L H, Gao Q M, Tan Y L, Tian W Q, Xu J D, Yang K et al 2015 Microporous Mesoporous Mater. 2101

[37] Luan Y T, Wang L, Guo S, Jiang B J, Zhao D D, Yan H J et al 2015 RSC Adv. 542430

[38] Zhao J, Lai H, Lyu Z, Jiang Y, Xie K, Wang X et al 2015 Adv. Mater. 273541

[39] Yoon Y, Lee K, Kwon S, Seo S, Yoo H, Kim S et al 2014 ACS Nano 84580 